ESAIM: M2AN

Vol. 40, No 2, 2006, pp. 353-366

DOI: $10.1051 / \mathrm{m} 2 \mathrm{an}: 2006016$
ESAIM: Mathematical Modelling and Numerical Analysis

www.edpsciences.org/m2an

\title{
NUMERICAL ANALYSIS OF THE QUASISTATIC THERMOVISCOELASTIC THERMISTOR PROBLEM*
}

\author{
José R. FERnÁNDEZ ${ }^{1}$
}

\begin{abstract}
In this work, the quasistatic thermoviscoelastic thermistor problem is considered. The thermistor model describes the combination of the effects due to the heat, electrical current conduction and Joule's heat generation. The variational formulation leads to a coupled system of nonlinear variational equations for which the existence of a weak solution is recalled. Then, a fully discrete algorithm is introduced based on the finite element method to approximate the spatial variable and an Euler scheme to discretize the time derivatives. Error estimates are derived and, under suitable regularity assumptions, the linear convergence of the scheme is deduced. Finally, some numerical simulations are performed in order to show the behaviour of the algorithm.
\end{abstract}

Mathematics Subject Classification. 65N15, 65N30, 74D10, 74S05, 74S20.

Received: June 13, 2005.

\section{INTRODUCTION}

During the last twenty years, the thermistor problem has been studied by many authors (see, e.g., $[1-4,8,10,14,15,21,22,25])$, dealing with different issues such as the existence and uniqueness of weak solutions or the degenerate cases, the so-called capacity solutions. Recently, in $[5,16-19,26]$ the numerical solution of the thermistor problem has been considered, under different mechanical conditions, using finite differences or the finite element method.

The thermistor model describes the combination of the effects due to the heat, electrical current conduction and Joule's heat generation. The electrical conductivity is assumed increasing (see [20] for details concerning the physical setting). The novelty of this model is that thermoviscoelastic effects are taken into account. The existence of a unique weak solution for the dynamical problem was established in [20], and its proof is based on the regularization, time-retarding and a fixed point argument. This work continues [20] and it is parallel to [12], where a finite element algorithm is presented and numerical simulations are shown.

Here, our aim is to provide the numerical analysis for the quasistatic thermoviscoelastic thermistor problem. The paper is structured as follows. In Section 2, the mechanical problem and its variational formulation are

\footnotetext{
Keywords and phrases. Thermoviscoelastic thermistor, error estimates, finite elements, numerical simulations.

* This work was partially supported by MCYT-Spain (Project BFM2003-05357). It is also part of the project "New Materials, Adaptive systems and their Nonlinearities; Modelling, Control and Numerical Simulation" carried out in the framework of the European Community program "Improving the Human Research Potential and the Socio-Economic Knowledge Base" (Contract $n^{\circ}$ HPRN-CT-2002-00284).

1 Departamento de Matemática Aplicada, Facultade de Matemáticas, Campus Sur s/n, Universidade de Santiago de Compostela, 15782 Santiago de Compostela, Spain. jramon@usc.es
} 
presented following $[12,20]$. An existence and uniqueness result is recalled. Then, a fully discrete scheme is introduced in Section 3, using the finite element method to approximate the spatial variable and the backward Euler scheme to discretize the time derivatives. Error estimates are established on the approximative solutions and, as a consequence, the linear convergence of the algorithm is derived under suitable regularity assumptions. Finally, in Section 4 some two-dimensional examples are presented in order to show the performance of the method.

\section{MEChANICAL AND VARIATIONAL PROBLEMS}

In this section, we follow $[12,20]$ and we refer the reader there for details. Let $S^{d}$ denote the space of second order symmetric tensors defined on $\mathbb{R}^{d}$, and let ":" and $|\cdot|$ represent the inner product and the norm on $S^{d}$, respectively.

Let $\Omega \subset \mathbb{R}^{d}(d=2,3)$ be a bounded domain with a smooth boundary $\Gamma=\partial \Omega$, representing the isothermal reference configuration of the thermoviscoelastic body, the thermistor. We assume that $\Gamma$ is divided into two parts $\Gamma_{D}$ and $\Gamma_{N}$ such that $\Gamma_{D} \cap \Gamma_{N}=\emptyset$, where $\Gamma_{D}$ has positive measure. We denote by $\boldsymbol{\nu}=\left(\nu_{1}, \ldots, \nu_{d}\right)$ the outward unit normal vector to $\Gamma$.

The body is clamped on $\Gamma_{D}$ and moreover, the temperature and electrical potential are prescribed there. On $\Gamma_{N}$ the body is free, electrically insulated and exchanging heat with the environment. Dirichlet conditions are chosen on $\Gamma_{D}$ for the three fields, for the sake of simplicity. We let $\theta$ denote the temperature field, $\phi$ the electrical potential and $\boldsymbol{u}=\left(u_{1}, \ldots, u_{d}\right)$ the displacements field. Let $T>0$ and set $\Omega_{T}=\Omega \times(0, T)$.

The constitutive and motion equations are written in $\Omega_{T}$ as follows (see [11]):

$$
\begin{aligned}
& \rho c_{p} \frac{\partial \theta}{\partial t}-\frac{\partial}{\partial x_{j}}\left(k_{i j}(\theta) \frac{\partial \theta}{\partial x_{i}}\right)=\sigma_{e l}(\theta) \operatorname{Tr}\left(|\nabla \phi|^{2}\right)-m_{i j} \Theta_{r e f} \frac{\partial^{2} u_{i}}{\partial t \partial x_{j}} \\
& -\frac{\partial}{\partial x_{j}}\left(\sigma_{e l}(\theta) \frac{\partial \phi}{\partial x_{j}}\right)=0 \\
& -\frac{\partial}{\partial x_{j}}\left(\sigma_{i j}\right)=f_{i}, \\
& \sigma_{i j}=a_{i j k l} \frac{\partial u_{k}}{\partial x_{l}}+b_{i j k l} \frac{\partial^{2} u_{k}}{\partial t \partial x_{l}}-m_{i j} \theta .
\end{aligned}
$$

Here and below, $i, j, k, l=1, \ldots, d$ and summation over repeated indices is implied. $\operatorname{Tr}: \mathbb{R} \rightarrow \mathbb{R}$ is a truncation operator defined by

$$
\operatorname{Tr}(r)=\left\{\begin{array}{l}
r \quad \text { if } \quad|r| \leq L, \\
L \quad \text { if } \quad|r|>L,
\end{array}\right.
$$

where $L>0$ is a given constant assumed large enough. Finally, we note that the inertia term was neglected in the motion equation (3), and then the process is quasistatic.

Equation (1) is the energy equation expressed in terms of the temperature. Here, $\theta$ is measured with respect to a reference absolute temperature $\Theta_{\text {ref }}$, given in degrees Kelvin. We assume that $\Theta_{\text {ref }}$ is also the ambient temperature. The density $\rho(>0)$ and the heat capacity $c_{p}(>0)$ are assumed to be constant; $K=K(\theta)=$ $\left\{k_{i j}(\theta)\right\}$ and $M=\left\{m_{i j}\right\}$ are the heat conduction and thermal expansion tensors, respectively, and for the sake of generality it is assumed that the thermal and mechanical properties of the material are anisotropic. The electrical conductivity $\sigma_{e l}=\sigma_{e l}(\theta)$ is assumed to depend strongly on the temperature. However, we assume that the electrical properties of the material are isotropic.

Equation (2) represents the electrical charge conservation, and (4) is the material constitutive relation. Here, $A=\left\{a_{i j k l}\right\}$ is the elasticity tensor; $B=\left\{b_{i j k l}\right\}$ is the tensor of viscosity coefficients; $\boldsymbol{f}=\left(f_{1}, \ldots, f_{d}\right)$ represents the density of body forces. Moreover, if we denote by $\varepsilon$ the linearized strain tensor $\left(\varepsilon=\varepsilon(\boldsymbol{u})=\frac{1}{2}\left(\nabla \boldsymbol{u}+(\nabla \boldsymbol{u})^{T}\right)\right)$, 
we may write the constitutive equation (4) as

$$
\boldsymbol{\sigma}=A \varepsilon(\boldsymbol{u})+B \varepsilon\left(\frac{\partial \boldsymbol{u}}{\partial t}\right)-M \theta
$$

Although different possibilities for $\sigma_{e l}(\theta)$ have been considered in many publications (including the so-called "capacity solutions" in $[8,22-24])$, here it is assumed that the electrical conductivity is nondegenerate and bounded. Thus,

$$
0<\sigma_{*} \leq \sigma_{e l}(\theta) \leq \sigma^{*}
$$

for $\sigma_{*}$ sufficiently small, and some constant $\sigma^{*}$.

To complete the classical formulation of the problem we have to specify the initial and boundary conditions. For the sake of the simplicity, homogeneous conditions are assumed on $\Gamma_{D}$ for the displacement and temperature fields (but not for the electrical potential since it would imply that $\phi=0$ ), that is,

$$
\theta=0, \quad \phi=\phi_{b}, \quad \boldsymbol{u}=\mathbf{0} \quad \text { on } \Gamma_{D} \times[0, T] .
$$

On $\Gamma_{N}$ the body exchanges heat with the environment, it is electrically insulated and stress-free, thus,

$$
\begin{aligned}
-k_{i j} \frac{\partial \theta}{\partial x_{i}} \nu_{j} & =\alpha\left(\theta-\Theta_{\text {ref }}\right) \quad \text { on } \Gamma_{N} \times(0, T), \\
\frac{\partial \phi}{\partial x_{i}} \nu_{i} & =0 \quad \text { on } \Gamma_{N} \times(0, T), \\
\sigma_{i j} \nu_{j} & =0 \quad \text { on } \Gamma_{N} \times(0, T),
\end{aligned}
$$

where $\alpha$ is the convective heat transfer coefficient.

The initial conditions are,

$$
\theta=\theta^{0}, \quad \boldsymbol{u}=\boldsymbol{u}^{0} \quad \text { in } \Omega, \quad t=0,
$$

where $\theta^{0}, \boldsymbol{u}^{0}$ denote the initial temperature and the initial displacements, respectively.

The classical formulation of the quasistatic thermoviscoelastic thermistor problem is: find $\{\theta, \phi, \boldsymbol{u}\}$ such that (1)-(4), (6)-(10) hold.

We turn now to obtain a weak formulation of the problem. Let us define the following variational spaces:

$$
\begin{aligned}
& V=\left\{\eta \in H^{1}(\Omega) ; \eta=0 \text { on } \Gamma_{D}\right\} \\
& E=\left\{\boldsymbol{v} \in\left[H^{1}(\Omega)\right]^{d} ; \boldsymbol{v}=\mathbf{0} \text { on } \Gamma_{D}\right\}
\end{aligned}
$$

The following assumptions are done:

(i) The density $\rho$ and the heat capacity $c_{p}$ are positive constants.

(ii) The electrical conductivity $\sigma_{e l}$ satisfies (5) and it is Lipschitz continuous.

(iii) The thermal conductivity tensor $\left\{k_{i j}\right\}$ is bounded and Lipschitz continuous, satisfying

$$
k_{i j} \xi_{i} \xi_{j} \geq \delta|\boldsymbol{\xi}|^{2}
$$

for all vectors $\boldsymbol{\xi}=\left(\xi_{i}\right)_{i=1}^{d} \in \mathbb{R}^{d}$ and some positive $\delta$.

(iv) The elasticity and viscosity tensors $\left\{a_{i j k l}\right\}$ and $\left\{b_{i j k l}\right\}$ are bounded and Lipschitz continuous, satisfying

$$
a_{i j k l} \tau_{i j} \tau_{k l} \geq \alpha_{1}|\tau|^{2}, \quad b_{i j k l} \tau_{i j} \tau_{k l} \geq \alpha_{2}|\tau|^{2},
$$

for all tensors $\boldsymbol{\tau}=\left(\tau_{i j}\right)_{i, j=1}^{d} \in S^{d}$ and some positive $\alpha_{1}, \alpha_{2}$.

(v) The initial temperature and the initial displacements satisfy $\theta^{0} \in V$ and $\boldsymbol{u}^{0} \in E$, respectively. 
(vi) The density of body forces has the regularity $\boldsymbol{f} \in C\left([0, T] ;\left[L^{2}(\Omega)\right]^{d}\right)$.

(vii) The function $\phi_{b} \in C\left([0, T] ; W^{1,4}(\Omega)\right)$.

The variational form of (1)-(4), (6)-(10) is: find $\theta:[0, T] \longrightarrow V, \phi:[0, T] \longrightarrow H^{1}(\Omega)$ and $\boldsymbol{u}, \boldsymbol{v}:[0, T] \longrightarrow E$ such that, for a.e. $t \in[0, T]$,

$$
\begin{aligned}
& \phi(t)-\phi_{b}(t) \in V, \quad \int_{\Omega} \sigma_{e l}(\theta(t)) \nabla \phi(t) \cdot \nabla r \mathrm{~d} \boldsymbol{x}=0, \quad \forall r \in V, \\
& \left\langle\rho c_{p} \frac{\partial \theta}{\partial t}(t), z\right\rangle_{V^{\prime} V}+\int_{\Omega} k_{i j}(\theta(t)) \frac{\partial \theta}{\partial x_{i}}(t) \frac{\partial z}{\partial x_{j}} \mathrm{~d} \boldsymbol{x}+\int_{\Gamma_{N}} \alpha\left(\theta(t)-\Theta_{r e f}\right) z \mathrm{~d} \Gamma \\
& \left.=\int_{\Omega} \sigma_{e l}(\theta(t)) \operatorname{Tr}\left(|\nabla \phi(t)|^{2}\right)\right) z \mathrm{~d} \boldsymbol{x}-\int_{\Omega} \Theta_{r e f} M: \boldsymbol{\varepsilon}(\boldsymbol{v}(t)) z \mathrm{~d} \boldsymbol{x}, \forall z \in V, \\
& \int_{\Omega}[B \boldsymbol{\varepsilon}(\boldsymbol{v}(t))+A \boldsymbol{\varepsilon}(\boldsymbol{u}(t))]: \boldsymbol{\varepsilon}(\boldsymbol{w}) \mathrm{d} \boldsymbol{x}=\int_{\Omega} \boldsymbol{f}(t) \cdot \boldsymbol{w} \mathrm{d} \boldsymbol{x}+\int_{\Omega} M \theta(t): \boldsymbol{\varepsilon}(\boldsymbol{w}) \mathrm{d} \boldsymbol{x}, \quad \forall \boldsymbol{w} \in E, \\
& \boldsymbol{u}(t)=\boldsymbol{u}^{0}+\int_{0}^{t} \boldsymbol{v}(s) \mathrm{d} s .
\end{aligned}
$$

An existence result for a dynamic version of problem (13)-(16) was given in [20]. It is based on time-retarded approximate problems, a priori estimates and compactness arguments. That proof can be modified in order to obtain the existence of a solution to problem (13)-(16), which we summarize in the following.

Theorem 1.1. Let the assumptions (i)-(vii) hold. Then, there exists a solution $\{\boldsymbol{u}, \theta, \phi\}$ to problem (13)-(16) with the following regularity:

$$
\boldsymbol{u} \in H^{1}(0, T ; E), \quad \phi \in L^{2}(0, T ; V), \quad \theta \in L^{2}(0, T ; V) \cap H^{1}\left(0, T ; V^{\prime}\right) .
$$

Remark 1.2. The regularity (17) does not allow to obtain the error estimates provided in the next section. However, since in this case the problem is quasistatic and the quadratic term in the heat equation was replaced by a Lipschitz function, it seems reasonable to assume that this regularity can be improved using, for instance, Banach fixed point arguments. This problem remains an open question and it will be addressed in the future.

\section{A FULLY DisCRETE SCHEME: ERROR ESTIMATES}

In this section, the above variational problem is discretized in time using the backward Euler scheme and in space by a finite element method. The functions $\boldsymbol{u}, \phi$ and $\theta$ are approximated by continuous piecewise affine functions on a defined mesh. Thus, we obtain a linear discrete problem for the displacement equation coupled with a nonlinear discrete problem for the temperature and the electrical potential which is solved by an iterative algorithm.

For the time discretization, we denote by $N$ the number of time steps and by $\Delta t=T / N$ the time step. For a continuous function $F$, let $F^{n}=F\left(t_{n}\right)$ with $t_{n}=n \Delta t$, for $n=0, \ldots, N$.

Associated with a family of regular meshes $\mathcal{T}_{h}$ of the domain $\Omega$, we consider the finite element spaces $E_{h}$ and $V_{h}$ given by

$$
\begin{aligned}
& E_{h}=\left\{\boldsymbol{v}_{h} \in[C(\bar{\Omega})]^{d} ; \boldsymbol{v}_{\left.h\right|_{K}} \in\left[P_{1}(K)\right]^{d}, \quad \forall K \in \mathcal{T}_{h}, \quad \boldsymbol{v}_{\left.h\right|_{\Gamma_{D}}}=\mathbf{0}\right\}, \\
& V_{h}=\left\{r_{h} \in C(\bar{\Omega}) ; r_{\left.h\right|_{K}} \in P_{1}(K), \quad \forall K \in \mathcal{T}_{h}, \quad r_{\left.h\right|_{\Gamma_{D}}}=0\right\},
\end{aligned}
$$

where $P_{1}(K)$ denotes the space of polynomials of global degree less or equal 1 defined in an element $K$. 
In order to simplify the writing and the calculations presented below, let us assume that $\phi_{b}=0$. It is straightforward to extend the error estimates to the nonhomogeneous case by using the orthogonal projection operator over $V_{h}$.

Then, using the backward Euler scheme to discretize the time derivatives, the following fully discretized problem is introduced: Find $\boldsymbol{u}_{h}=\left\{\boldsymbol{u}_{h}^{n}\right\}_{n=0}^{N}, \boldsymbol{v}_{h}=\left\{\boldsymbol{v}_{h}^{n}\right\}_{n=0}^{N} \subset E_{h}$ and $\theta_{h}=\left\{\theta_{h}^{n}\right\}_{n=0}^{N}, \phi_{h}=\left\{\phi_{h}^{n}\right\}_{n=0}^{N} \subset V_{h}$ such that $\boldsymbol{u}_{h}^{0}=\boldsymbol{u}^{0, h}, \theta_{h}^{0}=\theta^{0, h}$ and for $n=1, \ldots, N$,

$$
\begin{aligned}
& \int_{\Omega}\left[B \boldsymbol{\varepsilon}\left(\boldsymbol{v}_{h}^{n}\right)+\Delta t A \varepsilon\left(\boldsymbol{v}_{h}^{n}\right)\right]: \boldsymbol{\varepsilon}\left(\boldsymbol{w}_{h}\right) \mathrm{d} \boldsymbol{x}=\int_{\Omega} \boldsymbol{f}^{n} \cdot \boldsymbol{w}_{h} \mathrm{~d} \boldsymbol{x} \\
& \quad+\int_{\Omega}\left[M \theta_{h}^{n-1}-A \boldsymbol{\varepsilon}\left(\boldsymbol{u}_{h}^{n-1}\right)\right]: \boldsymbol{\varepsilon}\left(\boldsymbol{w}_{h}\right) \mathrm{d} \boldsymbol{x}, \quad \forall \boldsymbol{w}_{h} \in E_{h}, \\
& \boldsymbol{u}_{h}^{n}=\boldsymbol{u}_{h}^{n-1}+\Delta t \boldsymbol{v}_{h}^{n}, \\
& \int_{\Omega} \sigma_{e l}\left(\theta_{h}^{n}\right) \nabla \phi_{h}^{n} \cdot \nabla r_{h} \mathrm{~d} \boldsymbol{x}=0, \quad \forall r_{h} \in V_{h}, \\
& \frac{1}{\Delta t} \int_{\Omega} \rho c_{p} \theta_{h}^{n} z_{h} \mathrm{~d} \boldsymbol{x}+\int_{\Omega} k_{i j}\left(\theta_{h}^{n}\right) \frac{\partial \theta_{h}^{n}}{\partial x_{i}} \frac{\partial z_{h}}{\partial x_{j}} \mathrm{~d} \boldsymbol{x}+\int_{\Gamma_{N}} \alpha \theta_{h}^{n} z_{h} \mathrm{~d} \Gamma \\
& =\frac{1}{\Delta t} \int_{\Omega} \rho c_{p} \theta_{h}^{n-1} z_{h} \mathrm{~d} \boldsymbol{x}+\int_{\Omega} \sigma_{e l}\left(\theta_{h}^{n}\right) \operatorname{Tr}\left(\left|\nabla \phi_{h}^{n}\right|^{2}\right) z_{h} \mathrm{~d} \boldsymbol{x}+\int_{\Gamma_{N}} \alpha \Theta_{r e f} z_{h} \mathrm{~d} \Gamma \\
& \quad-\int_{\Omega} \Theta_{r e f} M: \varepsilon\left(\boldsymbol{v}_{h}^{n}\right) z_{h} \mathrm{~d} \boldsymbol{x}, \quad \forall z_{h} \in V_{h},
\end{aligned}
$$

where $\boldsymbol{u}^{0, h} \in E_{h}$ and $\theta^{0, h} \in V_{h}$ are appropriate approximations of the initial conditions $\boldsymbol{u}^{0}$ and $\theta^{0}$, respectively.

Remark 2.1. Notice that the discrete problem (20) can be seen as a linear system which is solved using Cholesky's method and then, displacements are updated from (21). Besides, discretized problem (22)-(23) is a nonlinear system due to the temperature dependence on the physical parameters, as the electrical and thermal conductivities, and the Joule's source term in (23). It is solved using a penalty-duality algorithm, coupled with fixed point iterations, introduced in [6].

The aim of this paper is to obtain an error estimates for the energy norms $\left\|\theta_{h}^{n}-\theta^{n}\right\|_{V},\left\|\phi_{h}^{n}-\phi^{n}\right\|_{V}$ and $\left\|\boldsymbol{u}_{h}^{n}-\boldsymbol{u}^{n}\right\|_{E}$. Then, let us assume the following regularity conditions on the continuous solution,

$$
\begin{aligned}
& \phi \in C\left([0, T] ; H^{1}(\Omega) \cap W^{1, \infty}(\Omega)\right), \quad \boldsymbol{u} \in C^{1}\left([0, T] ;\left[H^{1}(\Omega)\right]^{d}\right), \\
& \theta \in C\left([0, T] ; H^{1}(\Omega) \cap W^{1, \infty}(\Omega)\right) \cap C^{1}\left([0, T] ; L^{2}(\Omega)\right) .
\end{aligned}
$$

Remark 2.2. We note that there is a gap between these assumptions, required for obtaining the error estimates presented below, and the regularity of the solution provided in Theorem 1.1. This gap is an open problem that needs to be addressed.

First, we write (13) at time $t=t_{n}$. Taking $r=r_{h} \in V_{h} \subset V$ and substracting it to (22) we find that

$$
\int_{\Omega}\left[\sigma_{e l}\left(\theta^{n}\right) \nabla \phi^{n}-\sigma_{e l}\left(\theta_{h}^{n}\right) \nabla \phi_{h}^{n}\right] \cdot \nabla r_{h} \mathrm{~d} \boldsymbol{x}=0, \quad \forall r_{h} \in V_{h},
$$

and therefore,

$$
\int_{\Omega}\left[\sigma_{e l}\left(\theta^{n}\right) \nabla \phi^{n}-\sigma_{e l}\left(\theta_{h}^{n}\right) \nabla \phi_{h}^{n}\right] \cdot \nabla\left(\phi^{n}-\phi_{h}^{n}\right) \mathrm{d} \boldsymbol{x}=\int_{\Omega}\left[\sigma_{e l}\left(\theta^{n}\right) \nabla \phi^{n}-\sigma_{e l}\left(\theta_{h}^{n}\right) \nabla \phi_{h}^{n}\right] \cdot \nabla\left(\phi^{n}-r_{h}\right) \mathrm{d} \boldsymbol{x}, \quad \forall r_{h} \in V_{h} .
$$


Now, keeping in mind that

$$
\int_{\Omega}\left[\sigma_{e l}\left(\theta^{n}\right) \nabla \phi^{n}-\sigma_{e l}\left(\theta_{h}^{n}\right) \nabla \phi_{h}^{n}\right] \cdot \nabla r \mathrm{~d} \boldsymbol{x}=\int_{\Omega}\left[\left(\sigma_{e l}\left(\theta^{n}\right)-\sigma_{e l}\left(\theta_{h}^{n}\right)\right) \nabla \phi^{n}+\sigma_{e l}\left(\theta_{h}^{n}\right)\left(\nabla \phi^{n}-\nabla \phi_{h}^{n}\right)\right] \cdot \nabla r \mathrm{~d} \boldsymbol{x},
$$

for all $r \in V$, and using the regularity $\phi^{n} \in W^{1, \infty}(\Omega)$, property (ii) and the Cauchy's inequality

$$
a b \leq \varepsilon a^{2}+\frac{1}{4 \varepsilon} b^{2}, \quad a, b, \varepsilon>0,
$$

after easy algebraic manipulations we have, for all $r_{h}^{n} \in V_{h}$,

$$
\begin{aligned}
c\left\|\phi^{n}-\phi_{h}^{n}\right\|_{V}^{2} \leq & -\int_{\Omega}\left(\sigma_{e l}\left(\theta^{n}\right)-\sigma_{e l}\left(\theta_{h}^{n}\right)\right) \nabla \phi^{n} \cdot \nabla\left(\phi^{n}-\phi_{h}^{n}\right) \mathrm{d} \boldsymbol{x} \\
& +\int_{\Omega}\left(\sigma_{e l}\left(\theta^{n}\right)-\sigma_{e l}\left(\theta_{h}^{n}\right)\right) \nabla \phi^{n} \cdot \nabla\left(\phi^{n}-r_{h}^{n}\right) \mathrm{d} \boldsymbol{x} \\
& +\int_{\Omega} \sigma_{e l}\left(\theta_{h}^{n}\right)\left(\nabla \phi^{n}-\nabla \phi_{h}^{n}\right) \cdot \nabla\left(\phi^{n}-r_{h}^{n}\right) \mathrm{d} \boldsymbol{x} \\
\leq & c\left(\left\|\phi^{n}-r_{h}^{n}\right\|_{V}^{2}+\left\|\theta^{n}-\theta_{h}^{n}\right\|_{L^{2}(\Omega)}^{2}+\varepsilon\left\|\phi^{n}-\phi_{h}^{n}\right\|_{V}^{2}\right),
\end{aligned}
$$

where $\varepsilon$ is assumed to be small enough and $c>0$, here and everywhere below, is a positive constant that may vary from line to line and it is independent of the discretization parameters $h$ and $\Delta t$.

Secondly, we rewrite the variational equation (15) at time $t=t_{n}$ for all $\boldsymbol{w}=\boldsymbol{w}_{h} \in E_{h} \subset E$ and we substract it to (20) to obtain

$$
\int_{\Omega}\left[B \varepsilon\left(\boldsymbol{v}^{n}\right)+A \varepsilon\left(\boldsymbol{u}^{n}\right)-B \varepsilon\left(\boldsymbol{v}_{h}^{n}\right)-A \varepsilon\left(\boldsymbol{u}_{h}^{n}\right)\right]: \boldsymbol{\varepsilon}\left(\boldsymbol{w}_{h}\right) \mathrm{d} \boldsymbol{x}-\int_{\Omega}\left[M \theta^{n}-M \theta_{h}^{n-1}\right]: \boldsymbol{\varepsilon}\left(\boldsymbol{w}_{h}\right) \mathrm{d} \boldsymbol{x}=0,
$$

where (21) was employed. Thus,

$$
\begin{aligned}
& \int_{\Omega}\left[B \boldsymbol{\varepsilon}\left(\boldsymbol{v}^{n}\right)+A \boldsymbol{\varepsilon}\left(\boldsymbol{u}^{n}\right)-B \boldsymbol{\varepsilon}\left(\boldsymbol{v}_{h}^{n}\right)-A \boldsymbol{\varepsilon}\left(\boldsymbol{u}_{h}^{n}\right)\right]: \boldsymbol{\varepsilon}\left(\boldsymbol{v}^{n}-\boldsymbol{v}_{h}^{n}\right) \mathrm{d} \boldsymbol{x}-\int_{\Omega}\left[M \theta^{n}-M \theta_{h}^{n-1}\right]: \boldsymbol{\varepsilon}\left(\boldsymbol{v}^{n}-\boldsymbol{v}_{h}^{n}\right) \mathrm{d} \boldsymbol{x} \\
& =\int_{\Omega}\left[B \boldsymbol{\varepsilon}\left(\boldsymbol{v}^{n}\right)+A \boldsymbol{\varepsilon}\left(\boldsymbol{u}^{n}\right)-B \boldsymbol{\varepsilon}\left(\boldsymbol{v}_{h}^{n}\right)-A \varepsilon\left(\boldsymbol{u}_{h}^{n}\right)\right]: \boldsymbol{\varepsilon}\left(\boldsymbol{v}^{n}-\boldsymbol{w}_{h}\right) \mathrm{d} \boldsymbol{x}-\int_{\Omega}\left[M \theta^{n}-M \theta_{h}^{n-1}\right]: \varepsilon\left(\boldsymbol{v}^{n}-\boldsymbol{w}_{h}\right) \mathrm{d} \boldsymbol{x}, \quad \forall \boldsymbol{w}_{h} \in E_{h} .
\end{aligned}
$$

Since

$$
\left\|\boldsymbol{u}^{n}-\boldsymbol{u}_{h}^{n}\right\|_{E}^{2} \leq c\left(\left\|\boldsymbol{u}^{0}-\boldsymbol{u}^{0, h}\right\|_{E}^{2}+\Delta t \sum_{j=1}^{n}\left\|\boldsymbol{v}^{j}-\boldsymbol{v}_{h}^{j}\right\|_{E}^{2}+I_{n}^{2}\right)
$$

where $I_{n}$ is the integration error given by

$$
I_{n}=\left\|\int_{0}^{t_{n}} \boldsymbol{v}(s) \mathrm{d} s-\Delta t \sum_{j=1}^{n} \boldsymbol{v}^{j}\right\|_{E}
$$

using now assumption (iv) and (25), after some algebra it follows that

$$
\begin{aligned}
\left\|\boldsymbol{v}^{n}-\boldsymbol{v}_{h}^{n}\right\|_{E}^{2} \leq c\left(\left\|\boldsymbol{v}^{n}-\boldsymbol{w}_{h}^{n}\right\|_{E}^{2}+\Delta t \sum_{j=1}^{n}\left\|\boldsymbol{v}^{j}-\boldsymbol{v}_{h}^{j}\right\|_{E}^{2}+\left\|\theta^{n-1}-\theta_{h}^{n-1}\right\|_{L^{2}(\Omega)}^{2}\right. & \\
& \left.+\left\|\theta^{n}-\theta^{n-1}\right\|_{L^{2}(\Omega)}^{2}+\left\|\boldsymbol{u}^{0}-\boldsymbol{u}^{0, h}\right\|_{E}^{2}+I_{n}^{2}\right), \quad \forall \boldsymbol{w}_{h}^{n} \in E_{h}
\end{aligned}
$$


Finally, we need to estimate the numerical errors on the temperature field. Then, we consider (14) at time $t=t_{n}$ for all $z=z_{h} \in V_{h} \subset V$. Substracting it to (23), it leads to the following variational equation,

$$
\begin{aligned}
\int_{\Omega} \rho c_{p}\left(\frac{\partial \theta}{\partial t}\right. & \left.\left(t_{n}\right)-\frac{\theta_{h}^{n}-\theta_{h}^{n-1}}{\Delta t}\right) z_{h} \mathrm{~d} \boldsymbol{x}+\int_{\Omega}\left[k_{i j}\left(\theta^{n}\right) \frac{\partial \theta}{\partial x_{i}}\left(t^{n}\right)-k_{i j}\left(\theta_{h}^{n}\right) \frac{\partial \theta_{h}^{n}}{\partial x_{i}}\right] \frac{\partial z_{h}}{\partial x_{j}} \mathrm{~d} \boldsymbol{x} \\
& \quad+\int_{\Omega} \Theta_{r e f} M: \boldsymbol{\varepsilon}\left(\boldsymbol{v}^{n}-\boldsymbol{v}_{h}^{n}\right) z_{h} \mathrm{~d} \boldsymbol{x}-\int_{\Omega}\left[\sigma_{e l}\left(\theta^{n}\right) \operatorname{Tr}\left(\left|\nabla \phi^{n}\right|^{2}\right)-\sigma_{e l}\left(\theta_{h}^{n}\right) \operatorname{Tr}\left(\left|\nabla \phi_{h}^{n}\right|^{2}\right)\right] z_{h} \mathrm{~d} \boldsymbol{x}=0, \quad \forall z_{h} \in V_{h},
\end{aligned}
$$

which implies

$$
\begin{aligned}
\int_{\Omega} \rho c_{p} & \left(\frac{\partial \theta}{\partial t}\left(t_{n}\right)-\frac{\theta_{h}^{n}-\theta_{h}^{n-1}}{\Delta t}\right)\left(\theta^{n}-\theta_{h}^{n}\right) \mathrm{d} \boldsymbol{x}+\int_{\Omega} \Theta_{r e f} M: \varepsilon\left(\boldsymbol{v}^{n}-\boldsymbol{v}_{h}^{n}\right)\left(\theta^{n}-\theta_{h}^{n}\right) \mathrm{d} \boldsymbol{x} \\
& +\int_{\Omega}\left[\left[k_{i j}\left(\theta^{n}\right) \frac{\partial \theta}{\partial x_{i}}\left(t^{n}\right)-k_{i j}\left(\theta_{h}^{n}\right) \frac{\partial \theta_{h}^{n}}{\partial x_{i}}\right] \frac{\partial\left(\theta^{n}-\theta_{h}^{n}\right)}{\partial x_{j}} \mathrm{~d} \boldsymbol{x}\right. \\
& -\int_{\Omega}\left[\sigma_{e l}\left(\theta^{n}\right) \operatorname{Tr}\left(\left|\nabla \phi^{n}\right|^{2}\right)-\sigma_{e l}\left(\theta_{h}^{n}\right) \operatorname{Tr}\left(\left|\nabla \phi_{h}^{n}\right|^{2}\right)\right]\left(\theta^{n}-\theta_{h}^{n}\right) \mathrm{d} \boldsymbol{x} \\
= & \int_{\Omega} \rho c_{p}\left(\frac{\partial \theta}{\partial t}\left(t_{n}\right)-\frac{\theta_{h}^{n}-\theta_{h}^{n-1}}{\Delta t}\right)\left(\theta^{n}-z_{h}\right) \mathrm{d} \boldsymbol{x}+\int_{\Omega} \Theta_{r e f} M: \varepsilon\left(\boldsymbol{v}^{n}-\boldsymbol{v}_{h}^{n}\right)\left(\theta^{n}-z_{h}\right) \mathrm{d} \boldsymbol{x} \\
& +\int_{\Omega}\left[k_{i j}\left(\theta^{n}\right) \frac{\partial \theta}{\partial x_{i}}\left(t^{n}\right)-k_{i j}\left(\theta_{h}^{n}\right) \frac{\partial \theta_{h}^{n}}{\partial x_{i}}\right] \frac{\partial\left(\theta^{n}-z_{h}\right)}{\partial x_{j}} \mathrm{~d} \boldsymbol{x} \\
& -\int_{\Omega}\left[\sigma_{e l}\left(\theta^{n}\right) \operatorname{Tr}\left(\left|\nabla \phi^{n}\right|^{2}\right)-\sigma_{e l}\left(\theta_{h}^{n}\right) \operatorname{Tr}\left(\left|\nabla \phi_{h}^{n}\right|^{2}\right)\right]\left(\theta^{n}-z_{h}\right) \mathrm{d} \boldsymbol{x}, \quad \forall z_{h} \in V_{h} .
\end{aligned}
$$

In order to simplify the writing, let us define $\delta \theta^{n}=\left(\theta^{n}-\theta^{n-1}\right) / \Delta t$ and $\delta \theta_{h}^{n}=\left(\theta_{h}^{n}-\theta_{h}^{n-1}\right) / \Delta t$. Using the properties (i), (ii) and (iii), the Cauchy's inequality (25) and the regularity $\theta^{n} \in W^{1, \infty}(\Omega)$, and keeping in mind that

$$
\begin{aligned}
k_{i j}\left(\theta^{n}\right) \frac{\partial \theta}{\partial x_{i}}\left(t^{n}\right)-k_{i j}\left(\theta_{h}^{n}\right) \frac{\partial \theta_{h}^{n}}{\partial x_{i}}= & \left(k_{i j}\left(\theta^{n}\right)-k_{i j}\left(\theta_{h}^{n}\right)\right) \frac{\partial \theta}{\partial x_{i}}\left(t_{n}\right)+k_{i j}\left(\theta_{h}^{n}\right)\left(\frac{\partial \theta}{\partial x_{i}}\left(t_{n}\right)-\frac{\partial \theta_{h}^{n}}{\partial x_{i}}\right), \\
\sigma_{e l}\left(\theta^{n}\right) \operatorname{Tr}\left(\left|\nabla \phi^{n}\right|^{2}\right)-\sigma_{e l}\left(\theta_{h}^{n}\right) \operatorname{Tr}\left(\left|\nabla \phi_{h}^{n}\right|^{2}\right)= & \left(\sigma_{e l}\left(\theta^{n}\right)-\sigma_{e l}\left(\theta_{h}^{n}\right)\right) \operatorname{Tr}\left(\left|\nabla \phi^{n}\right|^{2}\right) \\
& +\sigma_{e l}\left(\theta_{h}^{n}\right)\left(\operatorname{Tr}\left(\left|\nabla \phi^{n}\right|^{2}\right)-\operatorname{Tr}\left(\left|\nabla \phi_{h}^{n}\right|^{2}\right)\right),
\end{aligned}
$$

after easy algebraic manipulations we find

$$
\begin{aligned}
\int_{\Omega}\left(\delta \theta^{n}-\delta \theta_{h}^{n}\right) & \left(\theta^{n}-\theta_{h}^{n}\right) \mathrm{d} \boldsymbol{x}+c\left\|\theta^{n}-\theta_{h}^{n}\right\|_{V}^{2} \leq c\left(\left\|\frac{\partial \theta}{\partial t}\left(t_{n}\right)-\delta \theta^{n}\right\|_{L^{2}(\Omega)}^{2}\right. \\
+ & \varepsilon\left\|\theta^{n}-\theta_{h}^{n}\right\|_{V}^{2}+\left\|\boldsymbol{v}^{n}-\boldsymbol{v}_{h}^{n}\right\|_{E}^{2}+\left\|\theta^{n}-\theta_{h}^{n}\right\|_{L^{2}(\Omega)}^{2}+\left\|\phi^{n}-\phi_{h}^{n}\right\|_{V}^{2} \\
& \left.+\left\|\theta^{n}-z_{h}^{n}\right\|_{V}^{2}+\int_{\Omega}\left(\delta \theta^{n}-\delta \theta_{h}^{n}\right)\left(\theta^{n}-z_{h}^{n}\right) \mathrm{d} \boldsymbol{x}\right), \quad \forall z_{h}^{n} \in V_{h},
\end{aligned}
$$

where $\varepsilon>0$ is assumed to be sufficiently small.

Since

$$
\int_{\Omega}\left(\delta \theta^{n}-\delta \theta_{h}^{n}\right)\left(\theta^{n}-\theta_{h}^{n}\right) \mathrm{d} \boldsymbol{x} \geq \frac{1}{2 \Delta t}\left[\left\|\theta^{n}-\theta_{h}^{n}\right\|_{L^{2}(\Omega)}^{2}-\left\|\theta^{n-1}-\theta_{h}^{n-1}\right\|_{L^{2}(\Omega)}^{2}\right]
$$


proceeding by induction we obtain

$$
\begin{aligned}
\left\|\theta^{n}-\theta_{h}^{n}\right\|_{L^{2}(\Omega)}^{2}+\Delta t \sum_{j=1}^{n}\left\|\theta^{j}-\theta_{h}^{j}\right\|_{V}^{2} \leq c \Delta t \sum_{j=1}^{n}\left(\left\|\frac{\partial \theta}{\partial t}\left(t_{j}\right)-\delta \theta^{j}\right\|_{L^{2}(\Omega)}^{2}\right. \\
\left.+\left\|\boldsymbol{v}^{j}-\boldsymbol{v}_{h}^{j}\right\|_{E}^{2}+\left\|\theta^{j}-\theta_{h}^{j}\right\|_{L^{2}(\Omega)}^{2}+\left\|\phi^{j}-\phi_{h}^{j}\right\|_{V}^{2}+\left\|\theta^{j}-z_{h}^{j}\right\|_{V}^{2}\right) \\
+c\left\|\theta^{0}-\theta^{0, h}\right\|_{L^{2}(\Omega)}^{2}+c \sum_{j=1}^{n} \int_{\Omega}\left(\theta^{j}-\theta^{j-1}-\left(\theta_{h}^{j}-\theta_{h}^{j-1}\right)\right)\left(\theta^{j}-z_{h}^{j}\right) \mathrm{d} \boldsymbol{x}, \quad\left\{z_{h}^{j}\right\}_{j=1}^{n} \subset V_{h} .
\end{aligned}
$$

Using similar ideas to those applied in [7] for estimating the numerical errors in the damage field, we have

$$
\begin{aligned}
\sum_{j=1}^{n} \int_{\Omega}\left(\theta^{j}-\theta^{j-1}-\left(\theta_{h}^{j}-\theta_{h}^{j-1}\right)\right)\left(\theta^{j}-z_{h}^{j}\right) \mathrm{d} \boldsymbol{x}=\int_{\Omega}\left(\theta^{n}-\theta_{h}^{n}\right)\left(\theta^{n}-z_{h}^{n}\right) \mathrm{d} \boldsymbol{x}+\int_{\Omega}\left(\theta^{0}-\theta^{0, h}\right)\left(\theta^{1}-z_{h}^{1}\right) \mathrm{d} \boldsymbol{x} & \\
& +\sum_{j=1}^{n-1} \int_{\Omega}\left(\theta^{j}-\theta_{h}^{j}\right)\left(\theta^{j}-z_{h}^{j}-\left(\theta^{j+1}-z_{h}^{j+1}\right)\right) \mathrm{d} \boldsymbol{x},
\end{aligned}
$$

and then,

$$
\begin{aligned}
\left\|\theta^{n}-\theta_{h}^{n}\right\|_{L^{2}(\Omega)}^{2}+\Delta t \sum_{j=1}^{n}\left\|\theta^{j}-\theta_{h}^{j}\right\|_{V}^{2} \leq c \Delta t \sum_{j=1}^{n}\left(\left\|\frac{\partial \theta}{\partial t}\left(t_{j}\right)-\delta \theta^{j}\right\|_{L^{2}(\Omega)}^{2}\right. & \\
+ & \left.\left\|\boldsymbol{v}^{j}-\boldsymbol{v}_{h}^{j}\right\|_{E}^{2}+\left\|\theta^{j}-\theta_{h}^{j}\right\|_{L^{2}(\Omega)}^{2}+\left\|\phi^{j}-\phi_{h}^{j}\right\|_{V}^{2}+\left\|\theta^{j}-z_{h}^{j}\right\|_{V}^{2}\right) \\
+c\left\|\theta^{0}-\theta^{0, h}\right\|_{L^{2}(\Omega)}^{2}+c\left\|\theta^{n}-z_{h}^{n}\right\|_{L^{2}(\Omega)}^{2}+c\left\|\theta^{1}-z_{h}^{1}\right\|_{L^{2}(\Omega)}^{2} & \\
& +c \sum_{j=1}^{n-1} \int_{\Omega}\left(\theta^{j}-\theta_{h}^{j}\right)\left(\theta^{j}-z_{h}^{j}-\left(\theta^{j+1}-z_{h}^{j+1}\right)\right) \mathrm{d} \boldsymbol{x}, \quad \forall\left\{z_{h}^{j}\right\}_{j=1}^{n} \subset V_{h} .
\end{aligned}
$$

Let us denote by

$$
e_{n}=\left\|\theta^{n}-\theta_{h}^{n}\right\|_{L^{2}(\Omega)}^{2}+\Delta t \sum_{j=1}^{n}\left\|\theta^{j}-\theta_{h}^{j}\right\|_{V}^{2}+\left\|\boldsymbol{v}^{n}-\boldsymbol{v}_{h}^{n}\right\|_{E}^{2}+\left\|\phi^{n}-\phi_{h}^{n}\right\|_{V}^{2},
$$

for $n=1, \ldots, N$, and

the numerical errors, and define, for $n=1, \ldots, N$,

$$
e_{0}=\left\|\theta^{0}-\theta^{0, h}\right\|_{L^{2}(\Omega)}^{2}+\left\|\boldsymbol{u}^{0}-\boldsymbol{u}^{0, h}\right\|_{V}^{2}
$$

$$
\begin{aligned}
& g_{n}=\Delta t \sum_{j=1}^{n}\left(\left\|\frac{\partial \theta}{\partial t}\left(t_{j}\right)-\delta \theta^{j}\right\|_{L^{2}(\Omega)}^{2}+\left\|\theta^{j}-z_{h}^{j}\right\|_{V}^{2}\right)+\left\|\theta^{n}-z_{h}^{n}\right\|_{L^{2}(\Omega)}^{2} \\
&+\left\|\theta^{1}-z_{h}^{1}\right\|_{L^{2}(\Omega)}^{2}+\left\|\phi^{n}-r_{h}^{n}\right\|_{V}^{2}+\left\|\boldsymbol{v}^{n}-\boldsymbol{w}_{h}^{n}\right\|_{E}^{2}+\left\|\theta^{n}-\theta^{n-1}\right\|_{L^{2}(\Omega)}^{2} \\
& \quad+e_{0}+I_{n}^{2}+\sum_{j=1}^{n} \int_{\Omega}\left(\theta^{j}-\theta_{h}^{j}\right)\left(\theta^{j}-z_{h}^{j}-\left(\theta^{j+1}-z_{h}^{j+1}\right)\right) \mathrm{d} \boldsymbol{x},
\end{aligned}
$$

and

$$
g_{0}=e_{0}
$$


Combining now (26), (27) and (28), it leads to the following error estimates,

$$
e_{0} \leq g_{0}, \quad e_{n} \leq c\left(g_{n}+\Delta t \sum_{j=1}^{n} e_{j}\right), \quad \text { for } n=1, \ldots, N
$$

Finally, using a discrete version of Gronwall's lemma (see [13] for details), the following error estimates result is obtained.

Theorem 2.3. Let the assumptions (i)-(vii) and the regularity conditions (24) hold. Let $\{\boldsymbol{u}, \theta, \phi\}$ denote the solution to problem (13)-(16) and $\left\{\boldsymbol{u}_{h}, \theta_{h}, \phi_{h}\right\}$ the solution to discrete problem (20)-(23). Then, the following error estimates are obtained for all $\left\{z_{h}^{n}\right\}_{n=1}^{N} \subset V_{h},\left\{r_{h}^{n}\right\}_{n=1}^{N} \subset V_{h}$ and $\left\{\boldsymbol{w}_{h}^{n}\right\}_{n=1}^{N} \subset E_{h}$,

$$
\max _{0 \leq n \leq N}\left\{\left\|\theta^{n}-\theta_{h}^{n}\right\|_{L^{2}(\Omega)}^{2}+\left\|\boldsymbol{v}^{n}-\boldsymbol{v}_{h}^{n}\right\|_{E}^{2}+\left\|\phi^{n}-\phi_{h}^{n}\right\|_{V}^{2}\right\}+\Delta t \sum_{j=1}^{N}\left\|\theta^{j}-\theta_{h}^{j}\right\|_{V}^{2} \leq c \max _{0 \leq n \leq N} g_{n}
$$

where $g_{n}$ is given by (29)-(30).

We note that (31) is the basis for the analysis of the convergence rate. Therefore, if we assume, for instance, that

$$
\begin{aligned}
& \theta \in H^{2}\left(0, T ; L^{2}(\Omega)\right) \cap C\left([0, T] ; H^{2}(\Omega)\right) \cap H^{1}\left(0, T ; H^{1}(\Omega)\right), \\
& \boldsymbol{u} \in C^{1}\left([0, T] ;\left[H^{2}(\Omega)\right]^{d}\right) \cap H^{2}\left(0, T ;\left[H^{1}(\Omega)\right]^{d}\right), \\
& \phi \in C\left([0, T] ; H^{2}(\Omega)\right),
\end{aligned}
$$

we have the following corollary which states the linear convergence of the numerical algorithm.

Corollary 2.4. Let the assumptions of Theorem 2.3 hold. Assume that the discrete initial conditions $\theta^{0, h}$ and $\boldsymbol{u}^{0, h}$ are given by

$$
\theta^{0, h}=\pi^{h}\left(\theta^{0}\right), \quad \boldsymbol{u}^{0, h}=\Pi^{h}\left(\boldsymbol{u}^{0}\right),
$$

where $\pi^{h}: C(\bar{\Omega}) \rightarrow V_{h}$ is the standard finite element interpolation operator (see [9]), and $\Pi^{h}=\left(\pi_{i}^{h}\right)_{i=1}^{d}$ : $[C(\bar{\Omega})]^{d} \rightarrow E_{h}$. Under the additional regularity conditions (32), the linear convergence of the fully discrete scheme is obtained; that is, there exists $c>0$, independent of $h$ and $\Delta t$, such that

$$
\max _{0 \leq n \leq N}\left\{\left\|\theta^{n}-\theta_{h}^{n}\right\|_{L^{2}(\Omega)}+\left\|\boldsymbol{u}^{n}-\boldsymbol{u}_{h}^{n}\right\|_{E}+\left\|\phi^{n}-\phi_{h}^{n}\right\|_{V}\right\} \leq c(h+\Delta t) .
$$

Proof. Since $\theta \in H^{2}\left(0, T ; L^{2}(\Omega)\right)$ it is easy to check that

$$
\sum_{j=1}^{N} \Delta t\left\|\frac{\partial \theta}{\partial t}\left(t_{j}\right)-\delta \theta^{j}\right\|_{L^{2}(\Omega)}^{2} \leq c(\Delta t)^{2}\|\theta\|_{H^{2}\left(0, T ; L^{2}(\Omega)\right)}^{2}
$$

and, using the approximation properties of the finite element spaces $V_{h}$ and $E_{h}$ and the regularity (32), it follows that (see [9])

$$
\begin{aligned}
& \max _{0 \leq n \leq N} \inf _{z_{h}^{n} \in V_{h}}\left\|\theta^{n}-z_{h}^{n}\right\|_{V}^{2} \leq c h^{2}\|\theta\|_{C\left([0, T] ; H^{2}(\Omega)\right)}^{2}, \\
& \max _{0 \leq n \leq N} \inf _{r_{h}^{n} \in V_{h}}\left\|\phi^{n}-r_{h}^{n}\right\|_{V}^{2} \leq c h^{2}\|\phi\|_{C\left([0, T] ; H^{2}(\Omega)\right),}^{2}, \\
& \max _{0 \leq n \leq N} \inf _{\boldsymbol{w}_{h}^{n} \in E_{h}}\left\|\boldsymbol{v}^{n}-\boldsymbol{w}_{h}^{n}\right\|_{E}^{2} \leq c h^{2}\|\boldsymbol{u}\|_{C^{1}\left([0, T] ;\left[H^{2}(\Omega)\right]^{d}\right)}^{2} .
\end{aligned}
$$


Keeping in mind that $\boldsymbol{u} \in H^{2}\left(0, T ;\left[H^{1}(\Omega)\right]^{d}\right)$ and $\theta \in H^{2}\left(0, T ; L^{2}(\Omega)\right)$, we have

$$
\begin{aligned}
I_{n}^{2} & \leq c(\Delta t)^{2}\|\boldsymbol{u}\|_{H^{2}\left(0, T ;\left[H^{1}(\Omega)\right]^{d}\right)}^{2}, \\
\max _{0 \leq n \leq N}\left\|\theta^{n}-\theta^{n-1}\right\|_{L^{2}(\Omega)}^{2} & \leq c(\Delta t)^{2}\|\theta\|_{H^{2}\left(0, T ; L^{2}(\Omega)\right)}^{2} .
\end{aligned}
$$

Since $\theta^{0, h}$ and $\boldsymbol{u}^{0, h}$ are defined by (33), well-known results (see [9] for details) lead to the following estimates:

$$
\begin{aligned}
& \left\|\theta^{0}-\theta^{0, h}\right\|_{L^{2}(\Omega)}^{2} \leq c h^{2}\left\|\theta^{0}\right\|_{H^{1}(\Omega)}^{2} \leq c h^{2}\|\theta\|_{C\left([0, T] ; H^{2}(\Omega)\right)}^{2}, \\
& \left\|\boldsymbol{u}^{0}-\boldsymbol{u}^{0, h}\right\|_{V}^{2} \leq c h^{2}\left\|\boldsymbol{u}^{0}\right\|_{\left[H^{2}(\Omega)\right]^{d}}^{2} \leq c h^{2}\|\boldsymbol{u}\|_{C^{1}\left([0, T] ;\left[H^{2}(\Omega)\right]^{d}\right)}^{2} .
\end{aligned}
$$

Finally, proceeding as in [7] we obtain that

$$
\sum_{j=1}^{N-1} \int_{\Omega}\left(\theta^{j}-\theta_{h}^{j}\right)\left(\theta^{j}-z_{h}^{j}-\left(\theta^{j+1}-z_{h}^{j+1}\right)\right) \mathrm{d} \boldsymbol{x} \leq c h^{2}\|\theta\|_{H^{1}\left(0, T ; H^{1}(\Omega)\right)}^{2},
$$

which concludes the proof.

\section{Numerical Simulations}

In order to verify the performance of the numerical algorithm described in the previous section and to gain insight into the behaviour of the solutions, we performed several numerical experiments. In this section we present two of the results obtained in these numerical simulations.

In all the problems presented below, the body is assumed to be under the plane stress hypothesis with elasticity tensor $A$,

$$
(A \boldsymbol{\tau})_{\alpha \beta}=\frac{E \kappa}{1-\kappa^{2}}\left(\tau_{11}+\tau_{22}\right) \delta_{\alpha \beta}+\frac{E}{1+\kappa} \tau_{\alpha \beta}, \quad \alpha, \beta=1,2, \quad \forall \boldsymbol{\tau}=\left(\tau_{\alpha \beta}\right) \in S^{2},
$$

where $\delta_{\alpha \beta}$ represents the Kronecker symbol, and $E$ and $\kappa$ are Young's modulus and Poisson's ratio of the material that occupies $\Omega$, respectively.

The viscosity tensor $B$ has a similar form,

$$
(B \boldsymbol{\tau})_{\alpha \beta}=\eta_{1}\left(\tau_{11}+\tau_{22}\right) \delta_{\alpha \beta}+\eta_{2} \tau_{\alpha \beta}, \quad \alpha, \beta=1,2, \quad \forall \boldsymbol{\tau}=\left(\tau_{\alpha \beta}\right) \in S^{2},
$$

where $\eta_{1}$ and $\eta_{2}$ are viscosity coefficients. Finally, the truncation value $L=1000$ is employed and the thermal expansion tensor $M$ is constant and homogeneous, namely $M=m I=\left\{m \delta_{\alpha \beta}\right\}$, with $m$ a positive constant.

\subsection{First example: convergence of the algorithm in an academical test}

As a first example we consider an academical test. We denote by $\Omega$ the unit square domain $(0,1) \times(0,1)$ which is the cross-section of a three-dimensional viscoelastic body. On the part $\Gamma_{D}=\{0,1\} \times[0,1]$ the body is clamped and so the displacement field vanishes there. The temperature field is also assumed to be fixed (and equal to $\left.\Theta_{r e f}\right)$ on $\Gamma_{D}$, and the electrical potential is defined by $\phi(x, y, t)=0$ if $x=0$ and $\phi(x, y, t)=1$ if $x=1$ for all $t \in[0,1](T=1 \mathrm{~s})$. Finally, the part $\Gamma_{N}$ is assumed traction-free, there is no heat exchange (value $\alpha=10^{9}$ is used for its simulation) and no body forces act in the thermistor.

In order to see the convergence behaviour of the scheme, a sequence of numerical solutions is computed based on uniform partitions of the time interval $[0,1]$, and uniform triangulations of the domain $[0,1] \times[0,1]$, where 
TABLE 1. Example 1: numerical errors (x100) for some $n$ and $\Delta t$.

\begin{tabular}{ccccccc}
\hline$n \downarrow \Delta t \rightarrow$ & 0.05 & 0.02 & 0.01 & 0.005 & 0.002 & 0.001 \\
\hline 4 & 3.971 & 3.971 & 3.971 & 3.971 & 3.971 & 3.971 \\
8 & 2.062 & 2.062 & 2.062 & 2.062 & 2.062 & 2.062 \\
16 & 1.039 & 1.039 & 1.039 & 1.039 & 1.039 & 1.039 \\
32 & 0.5102 & 0.5102 & 0.5102 & 0.5102 & 0.5102 & 0.5102 \\
64 & 0.2298 & 0.2298 & 0.2298 & 0.2298 & 0.2298 & 0.2298 \\
\hline
\end{tabular}

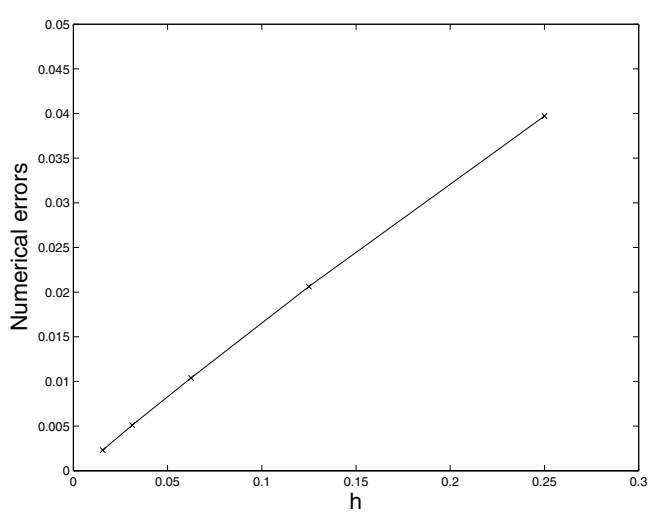

FiguRE 1. Example 1: asymptotic convergence with respect to $h$.

the interval $[0,1]$ is divided into $n$ equal parts. The following data are employed in the computations:

$$
\begin{aligned}
& T=1 \mathrm{~s}, \quad \Theta_{r e f}=273.15 \mathrm{~K}, \quad E=10 \mathrm{~N} / \mathrm{m}^{2}, \quad \kappa=0.3, \quad c_{p}=1 \mathrm{~J}, \\
& m=0.1 \mathrm{~N} /\left(\mathrm{m}^{2} \cdot \mathrm{K}\right), \quad \eta_{1}=1 \mathrm{~N} \cdot \mathrm{s} / \mathrm{m}^{2}, \quad \eta_{2}=1 \mathrm{~N} \cdot \mathrm{s} / \mathrm{m}^{2}, \quad \rho=1 \mathrm{~kg} / \mathrm{m}^{3}, \\
& \boldsymbol{f}=\mathbf{0 N} / \mathrm{m}^{3}, \quad \theta^{0}=273.15 \mathrm{~K}, \quad \boldsymbol{u}^{0}=\mathbf{0} \mathrm{m} .
\end{aligned}
$$

The electrical conductivity $\sigma_{e l}$ and the thermal conductivity tensor $k_{\alpha \beta}$ have the following form:

$$
\sigma_{e l}(\theta)=\theta \mathrm{s} / \mathrm{m}, \quad k_{\alpha \beta}(\theta)=2 \theta \delta_{\alpha \beta} \mathrm{W} / \mathrm{m} \cdot \mathrm{K}, \quad \alpha, \beta=1,2 .
$$

The numerical solution corresponding to $n=256(h \approx \sqrt{2} / n)$ and $\Delta t=0.0005$ is taken as the "exact solution", used to compute the numerical errors defined by the expression

$$
\max _{0 \leq n \leq N}\left\{\left\|\theta^{n}-\theta_{h}^{n}\right\|_{L^{2}(\Omega)}+\left\|\boldsymbol{u}^{n}-\boldsymbol{u}_{h}^{n}\right\|_{E}+\left\|\phi^{n}-\phi_{h}^{n}\right\|_{V}\right\} .
$$

In Table 1 these errors, multiplied by 100 and obtained for some $n$ and $\Delta t$, are shown. The numerical convergence is clearly observed. We also notice that there is not dependence on the time discretization parameter (the errors are less than $10^{-6}$ ). This is produced since the data are all time-independent. In Figure 1 the numerical errors are plotted with respect to the spatial discretization parameter $h$. The linear convergence is depicted.

If we consider now the following (time-dependent) boundary condition for the electrical potential,

$$
\phi(x, y, t)=\left\{\begin{array}{l}
10 e^{t} \quad \text { if } \quad x=0, y \in[0,1] \\
0 \quad \text { if } \quad x=1, y \in[0,1]
\end{array}\right.
$$

and we use the above data, in Table 2 the numerical errors are shown for some $n$ and $\Delta t$. Again, the numerical convergence is observed. However, the linear convergence rate is not achieved (see Fig. 2). 
TABLE 2. Example 1: numerical errors for some $n$ and $\Delta t$ (time-dependent boundary condition).

\begin{tabular}{ccccccc}
\hline$n \downarrow \Delta t \rightarrow$ & 0.05 & 0.02 & 0.01 & 0.005 & 0.002 & 0.001 \\
\hline 4 & 5.4235 & 3.8906 & 3.4233 & 3.2300 & 3.1402 & 3.1159 \\
8 & 3.6547 & 1.9360 & 1.3774 & 1.1262 & 1.0070 & 0.9767 \\
16 & 3.1960 & 1.4143 & 0.8126 & 0.5221 & 0.3717 & 0.3349 \\
32 & 3.0773 & 1.2794 & 0.6654 & 0.3586 & 0.1859 & 0.1372 \\
64 & 3.0451 & 1.2416 & 0.6243 & 0.3134 & 0.1283 & 0.0715 \\
\hline
\end{tabular}

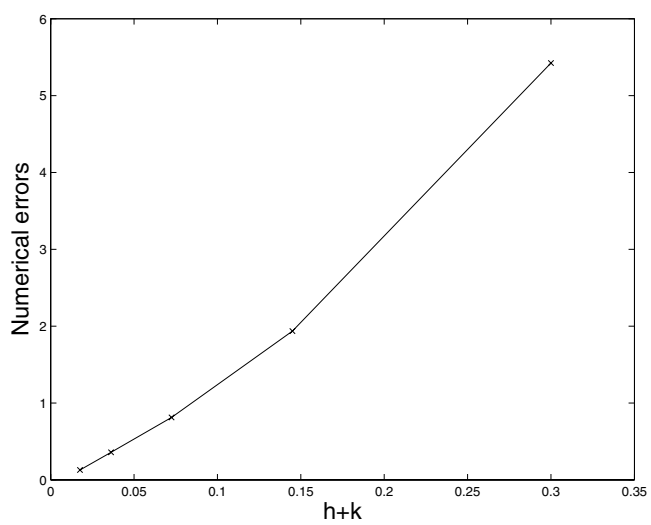

FiguRE 2. Example 1: asymptotic convergence with respect to $h+k$.

\subsection{Second example: influence of the heat transfer coefficient in a "real" thermistor}

As a second example, the setting is similar to the above one. The process is simulated during one second (i.e. $T=1 \mathrm{~s})$. The viscoelastic thermistor occupies the domain $\Omega=(0,1) \times(0,1)$ and it is assumed to be clamped on $\Gamma_{D}=\{0,1\} \times[0,1]$, while on $\Gamma_{N}=(0,1) \times\{0,1\}$ it is traction-free.

The following data are used in the simulations:

$$
\begin{aligned}
& T=1 \mathrm{~s}, \quad \Theta_{r e f}=273.15 \mathrm{~K}, \quad E=10^{9} \mathrm{~N} / \mathrm{m}^{2}, \quad \kappa=0.3, \quad c_{p}=20.83 \mathrm{~J} \\
& \boldsymbol{f}=\mathbf{0 ~ N} / \mathrm{m}^{3}, \quad \eta_{1}=5.76 \times 10^{3} \mathrm{~N} \cdot \mathrm{s} / \mathrm{m}^{2}, \quad \eta_{2}=3.84 \times 10^{3} \mathrm{~N} \cdot \mathrm{s} / \mathrm{m}^{2}, \\
& m=0.1 \mathrm{~N} /\left(\mathrm{m}^{2} \cdot \mathrm{K}\right), \quad \rho=2000 \mathrm{~kg} / \mathrm{m}^{3}, \quad \theta^{0}=273.15 \mathrm{~K}, \quad \boldsymbol{u}^{0}=\mathbf{0} \mathrm{m} .
\end{aligned}
$$

The heat conduction tensor is chosen as $k_{i j}(\theta)=\theta, i, j=1,2$, the electrical conductivity is given by

$$
\sigma_{e l}(\theta)=190-50\left(2+1.1 \tan ^{-1}(20(\theta-279.15))\right),
$$

and the following boundary conditions are defined for the temperature and electrical potential, respectively,

$$
\theta=273.15 \mathrm{~K} \quad \text { on } \quad \Gamma_{D}, \quad \phi(x, y, t)=\left\{\begin{array}{l}
100 \quad \text { if } x=0, y \in[0,1], \\
0 \quad \text { if } x=1, y \in[0,1] .
\end{array}\right.
$$

Finally, a finite element triangulation composed of 1073 nodes and 2024 triangles and a time discretization step $\Delta t=0.001$ are employed in the simulations.

The aim of this example is to investigate the influence of the parameter $\alpha$, the heat transfer coefficient, into the process. Therefore, in Figure 3 the temperature fields, corresponding to $\alpha=10$ and $\alpha=10000$, are plotted at final time. As we can see, increasing the value of $\alpha$ implies that the body exchanges less temperature, on the boundary $\Gamma_{N}$, with the environment. The corresponding electrical potentials and stress fields are shown, for the same values, in Figures 4 and 5, respectively. 

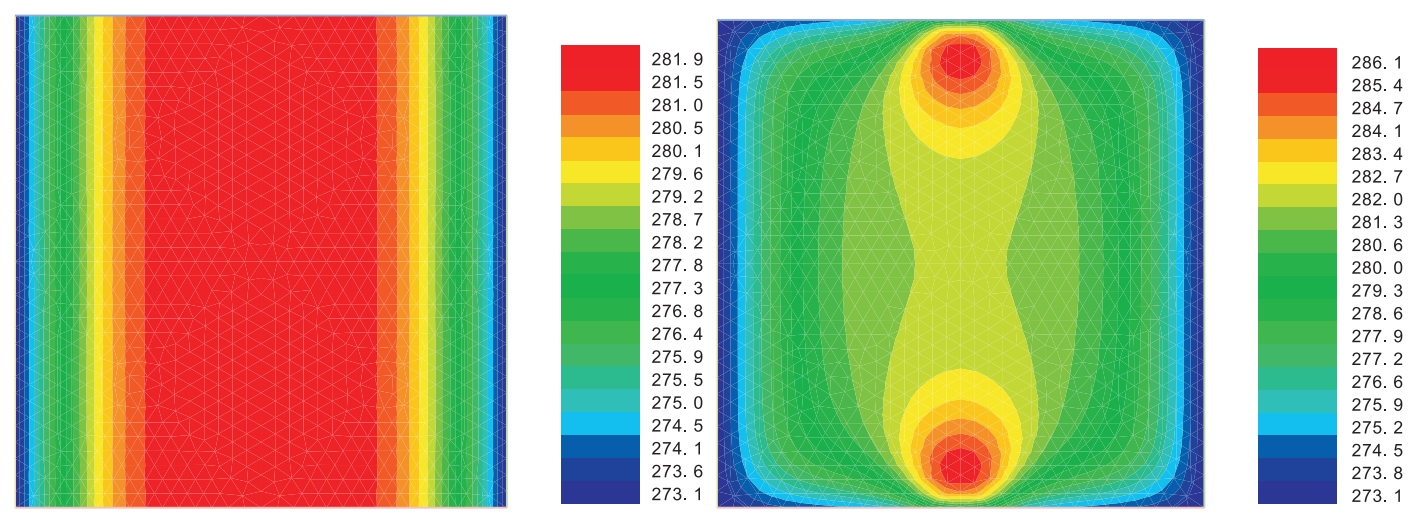

Figure 3. Example 2: temperature fields at final time for $\alpha=10,10000$.
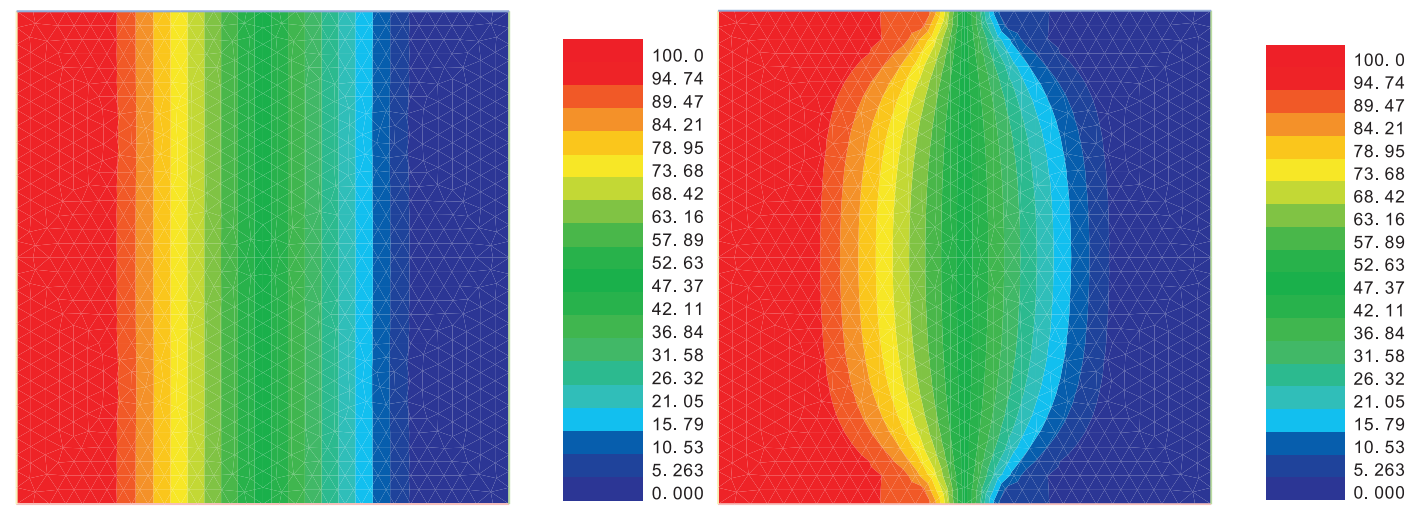

Figure 4. Example 2: electrical potentials at final time for $\alpha=10,10000$.
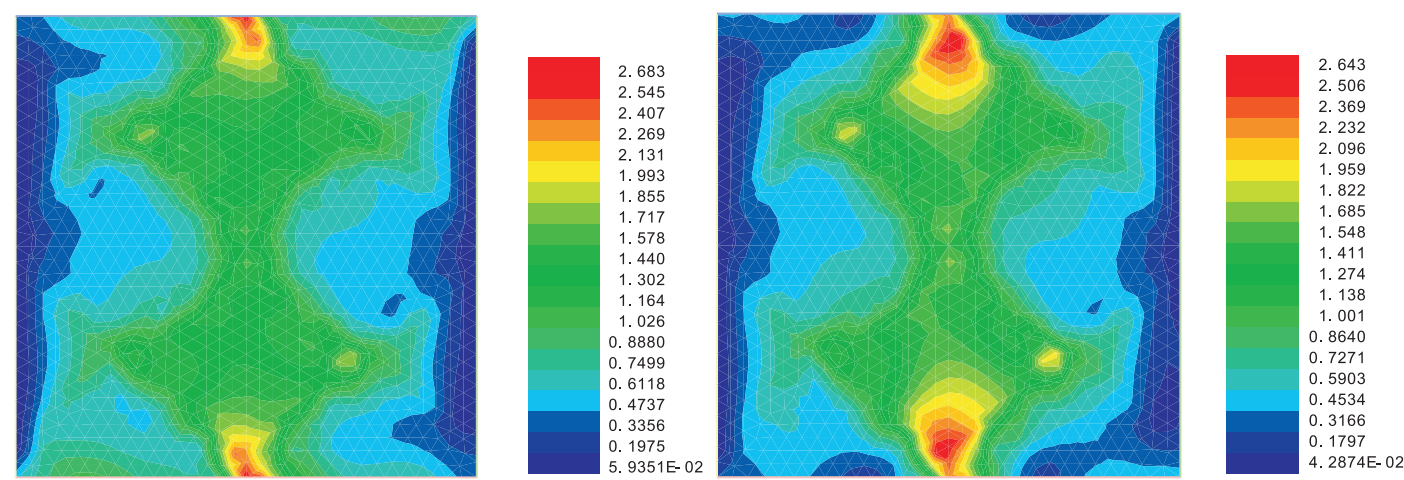

Figure 5. Example 2: stress fields at final time for $\alpha=10,10000$.

We notice that, when $\alpha$ increases, the electrical potential ceases to be constant with respect to the $Y$-variable. Moreover, stresses appear, in both cases, due to the increasing of the temperature, which may cause the breaking of the thermistor. 


\section{REFERENCES}

[1] W. Allegretto and H. Xie, A non-local thermistor problem. Eur. J. Appl. Math. 6 (1995) 83-94.

[2] W. Allegreto, Y. Lin and A. Zhou, A box scheme for coupled systems resulting from microsensor thermistor problems. Dynam. Contin. Discret. S. 5 (1999) 209-223.

[3] W. Allegreto, Y. Lin and S. Ma, Existence and long time behaviour of solutions to obstacle thermistor equations. Discrete Contin. Dyn. S. 8 (2002) 757-780.

[4] S.N. Antontsev and M. Chipot, The thermistor problem: existence, smoothness, uniqueness, blowup. SIAM J. Math. Anal. 25 (1994) 1128-1156.

[5] A.R. Bahadir, Application of cubic B-spline finite element technique to the thermistor problem. Appl. Math. Comput. 149 (2004) 379-387.

[6] A. Bermúdez, M.C. Muñiz and P. Quintela, Numerical solution of a three-dimensional thermoelectric problem taking place in an aluminum electrolytic cell. Comput. Method Appl. M. 106 (1993) 129-142.

[7] O. Chau, J.R. Fernández, W. Han and M. Sofonea, A frictionless contact problem for elastic-viscoplastic materials with normal compliance and damage. Comput. Method Appl. M. 191 (2002) 5007-5026.

[8] X. Chen, Existence and regularity of solutions of a nonlinear degenerate elliptic system arising from a thermistor problem. $J$. Partial Differential Equations 7 (1994) 19-34.

[9] P.G. Ciarlet, The finite element method for elliptic problems, in Handbook of Numerical Analysis, Vol. II, Part 1, P.G. Ciarlet and J.L. Lions Eds., North Holland (1991) 17-352.

[10] G. Cimatti, Remark on the existence and uniqueness for the thermistor problem under mixed boundary conditions. Quart. J. Mech. Appl. Math. 47 (1989) 117-121.

[11] G. Duvaut and J.L. Lions, Inequalities in Mechanics and Physics, Springer, New-York (1976).

[12] J.R. Fernández, K.L. Kuttler, M.C. Muñiz and M. Shillor, A model and simulations of the thermoviscoelastic thermistor. Eur. J. Appl. Math. (submitted).

[13] W. Han and M. Sofonea, Quasistatic contact problems in viscoelasticity and viscoplasticity, Americal Mathematical Society-International Press (2002).

[14] S.D. Howison, A note on the thermistor problem in two space dimension. Quart. J. Mech. Appl. Math. 47 (1989) 509-512.

[15] S.D. Howison, J. Rodrigues and M. Shillor, Stationary solutions to the thermistor problem. J. Math. Anal. Appl. 174 (1993) $573-588$.

[16] S. Kutluay, A.R. Bahadir and A. Ozdeć, A variety of finite difference methods to the thermistor with a new modified electrical conductivity. Appl. Math. Comput. 106 (1999) 205-213.

[17] S. Kutluay, A.R. Bahadir and A. Ozdeć, Various methods to the thermistor problem with a bulk electrical conductivity. Int. J. Numer. Method. Engrg. 45 (1999) 1-12.

[18] S. Kutluay and E. Esen, A B-spline finite element method for the thermistor problem with the modified electrical conductivity. Appl. Math. Comput. 156 (2004) 621-632.

[19] S. Kutluay and A.S. Wood, Numerical solutions of the thermistor problem with a ramp electrical conductivity. Appl. Math. Comput. 148 (2004) 145-162.

[20] K.L. Kuttler, M. Shillor and J.R. Fernández, Existence for the thermoviscoelastic thermistor problem. Differential Equations Dynam. Systems (to appear).

[21] H. Xie and W. Allegretto, $C^{\alpha}(\bar{\Omega})$ solutions of a class of nonlinear degenerate elliptic systems arising in the thermistor problem. SIAM J. Math. Anal. 22 (1991) 1491-1499.

[22] X. Xu, The thermistor problem with conductivity vanishing for large temperature. P. Roy. Soc. Edinb. A 124 (1994) 1-21.

[23] X. Xu, On the existence of bounded temperature in the thermistor problem with degeneracy. Nonlinear Anal. 42 (2000) 199-213.

[24] X. Xu, On the effects of thermal degeneracy in the thermistor problem. SIAM J. Math. Anal. 35 (4) (2003) 1081-1098.

[25] X. Xu, Local regularity theorems for the stationary thermistor problem. P. Roy. Soc. Edinb. A 134 (2004) 773-782.

[26] S. Zhou and D.R. Westbrook, Numerical solutions of the thermistor equations. J. Comput. Appl. Math. 79 (1997) 101-118. 